\title{
Determinación y caracterización botánica de hábitats mapuche del sectorZewko-Rüpükura comuna de Nueva Imperial (IX Región, Chile)
}

\author{
Carolyn Sánchez ${ }^{1}$, Enrique Hauenstein ${ }^{1}$ \& Luis Peralta ${ }^{2}$
}

\section{RESUMEN}

Se determinaron los hábitats mapuche presentes en la comunidad indígena Zewko del sector Rüpükura, comuna de Nueva Imperial, IX Región, Chile, y se caracterizaron botánicamente los más reconocidos. Se les analizó desde el punto de vista mapuche y occidental, aplicando paralelamente metodologías participativas y botánicas. Se identificaron 23 ecosistemas, de los cuales 8 fueron mencionados como los más relevantes; de éstos, el menoko (38\%), el malliñ (19\%) y el trayen (13\%) son los que presentaron los más altos porcentajes de importancia. Vegetacionalmente se caracterizaron dos comunidades vegetales nativas, la pradera húmeda de junquillo (Juncetum procerii), correspondiente al malliñ, y el bosque pantanoso de temo y pitra (Blepharocalyo-Myrceugenietum exsuccae), que corresponde al menoko. Con respecto a la flora vascular se identificaron 91 especies en el sector, de las cuales el $72 \%$ correspondió a especies nativas. La forma de vida mayormente representada fueron los hemicriptófitos con un 40\%. Al analizar los dos ámbitos de la investigación se reconoció la diferente concepción existente entre los sistemas de caracterización mapuche V/S el sistema ecológico occidental convencional, por lo que se hace esencial considerar estas diferencias a la hora de investigar acerca de los recursos naturales.

Palabras claves: hábitat, conocimiento mapuche, espacio natural, mapuche, Rüpükura.

Facultad de Ciencias, Universidad Católica de Temuco, Temuco, Chile. Casilla 15-D. ehauen@uct.cl

2 Centro de Desarrollo Sustentable, Universidad Católica de Temuco, Temuco, Chile. Casilla 15-D. 


\section{Revista CUHSO volumen 8 n 1}

\section{ABSTRACT}

The habitat "mapuche", which can be found in Zewko, mapuche comune, Rüpükura area, Nueva Imperial, IX region, Chile, were determined and the most common flora and vegetation in this area were identified. They were analyzed under an occidental and a mapuche perspective, using both botanical and participative metodologies to do so. 23 ecosystems were found and identified, 8 of these were mentioned as the most relevant. Of these 8 systems menoko (38\%), malliñ (19\%), trayen (13\%) reached the highest percentage. In addition to that, two kinds of plant communities were registered: rush wet meadow, (Juncetum procerii), and swampy wood of "temo und pitra" (Blepharocalyo-Myrceugenietum exsuccae). In the Zewko area 91 species belonging to vascular flora, of which $72 \%$ belong to native species. The most widespread life forms were hemicriptophytes with $40 \%$. When both aspects of the investigation were analyzed, it became clear that the system characterized by the mapuche and the conventional occidental system did not agree. It is very important to bear these differences in mind when an investigation about natural resources is made.

Key words: Habitat, mapuche knowledge, natural space, mapuche, Rüpükura.

\section{INTRODUCCIÓN}

Los distintos grupos humanos se han vinculado con su entorno tan armónicamente, que han podido desarrollar culturas donde la base de todas sus costumbres, tradiciones e historia es el ambiente en donde se mueven. Según Cohen (1976), la forma como vemos nuestro ambiente depende de lo que buscamos en él y la búsqueda está condicionada por la propia cultura, lo que provoca diferenciaciones entre los grupos humanos en su relación y acción con el medio.

Para los apaches, por ejemplo, el mundo estaba en continuo movimiento y muchos de sus rituales se centraban en elementos de la naturaleza como el aire y el viento, éstos a su vez formaban parte de las personas y de los seres minerales (http:// mapahumano.fiestras.com/).

Por su parte en la cosmovisión andina, el hombre no se siente por encima de la naturaleza, sino como parte de ella, existiendo con su entorno una relación de respeto y de íntima reciprocidad que es vivida ritualmente y que se expresa en la música, canto, danzas, leyendas, artesanía, etc. (http:// www.cantovivo.org). Asimismo, estos pueblos andinos se autodefinen en estrecha relación con su hábitat, de esta forma se encuentra el acapacha (nuestro mundo) dividido en tres sectores o niveles: los achachilas o mallcus, que son los espíritus de las montañas nevadas que circundan los pueblos; la Pachamama, que es la madre tierra, venerada como la siempre fértil madre universal que alimenta toda la vida del mundo y el amaru, que es la serpiente que se vincula con los ríos y canales de irrigación de las tierras agrícolas. Esta tripartición del espacio vital de los aymaras corresponde, desde el punto de vista económico y ecológico, a los siguientes niveles: 1) las cumbres de las montañas; 2) la cordillera en los niveles de pastoreo; 3) los valles y quebradas de la precordillera con la agricultura en terrazas regadas (Hidalgo et al., 1996).

En el caso de los guaraníes, toda su vida diaria está impregnada de experiencia mística y todo se relaciona con el mundo sobrenatural y con el natural, de forma que el carácter de los individuos se relaciona con su "alma animal". Así, un hombre nervioso tendrá un atsyygwa de mono y otro, enfadadizo, tendrá un atsyygwa de jaguar (http://mapahumano.fiestras.com/). 
Según Naikiai (1987), los shuar (uno de los grupos más numerosos de la amazonía ecuatoriana) consideran que cada ser o cada cosa tiene su «wakán». No puede existir una cosa que sea total y únicamente materia; cada árbol o animal es considerado como un ser, por lo que ellos tienen un respeto conciente del medio ambiente. Por su parte, los purépechas, desde los tiempos prehistóricos, desarrollaron un gran cúmulo de conocimiento acerca del universo vegetal donde estaban insertos (Toledo et al., 1980).

Del mismo modo, el pueblo mapuche ha vivido desde siglos en estrecha relación con su medio natural. A raíz de eso, ha desarrollado un profundo conocimiento de la vegetación que le rodea y una ecología cultural en que muchos aspectos de su cultura, desde su subsistencia hasta su espiritualidad, le vincula con la naturaleza (Bragg et al., 1986).

Según Loncon \& Martínez (1999), en la cultura mapuche la tierra es la madre de todas las vidas, es el lecho de los que viven y de los que ya murieron, el espacio donde habitan las fuerzas del bien y del mal. El bienestar en la tierra depende del equilibrio de las fuerzas que existen en ella, y para que esto suceda hay que ser respetuosos consigo mismo y con la naturaleza. La religiosidad se caracteriza por el valor y el respeto por la naturaleza. Los cerros, las aguas, los pozones, las piedras, los árboles y los animales interactúan cotidianamente con las personas. La naturaleza fue dejada por el Ser Supremo para que exista la vida y el bienestar de sus hijos.

Desde el punto de vista científico occidental, un ecosistema corresponde a comunidades de organismos que interactúan entre sí y su medio físico, como una sola unidad ecológica; por su parte, una comunidad corresponde a un grupo de organismos pertenecientes a varias especies distintas que concurren en el mismo hábitat o área y que interactúan mediante relaciones tróficas. Asimismo, el hábitat corresponde a una localidad, sitio y tipo particular de medio ambiente local ocupado por uno o varios organismos (Lincoln et al., 1995).

El presente estudio pretende caracterizar, desde el punto de vista mapuche, los distintos hábitat naturales reconocidos por la comunidad del sector Zewko-Rüpükura, y también desde el punto de vista occidental, considerando su flora y vegetación.

\section{METODOLOGÍA}

\section{Área de estudio}

El área de estudio corresponde a la comunidad indígena Zewko del sector Rüpükura, comuna de Nueva Imperial, Provincia de Cautín, IX Región, Chile (Fig 1). Se sitúa en las coordenadas 38²9'30" Latitud Sur y $72^{\circ} 45^{\prime} 30^{\prime \prime}$ Longitud Oeste, posee una superficie de 171,6 km+ y está a una distancia de $24 \mathrm{~km}$ de la ciudad de Chol-Chol; el principal curso de agua que recorre el sector es el río Pellahuén, el cual es afluente del río Chol-Chol.

Los suelos corresponden a la serie Nahuelbuta, la cual se caracteriza por presentar una topografía ondulada a montañosa, terrenos altos, roca metamórfica altamente micásea y material generador parcialmente intemperizado. Las capacidades de uso que caracterizan la zona son la III y la VII, los suelos de clase III presentan moderadas limitaciones en su uso y restringen la elección de cultivos. Aunque pueden ser buenas para ciertos cultivos, tienen severas limitaciones que reducen la elección de plantas o requieren de prácticas especiales de conservación o de ambas; a su vez, los suelos de clase VII poseen limitaciones muy severas que los hacen inadecuados para el cultivo. Su uso fundamental es pastoril y forestal. Respecto de sus características morfológicas, las pendientes que predominan son entre 15 y $30 \%$, lo que según MOPT (1992) correspondería a áreas montañosas de fuerte pendiente con escorrentía rápida o muy rápida (Peña, 1989).

Los manejos vegetacionales presentes en la comunidad son primeramente los de rotación de cultivo-pradera, los que corresponden a terrenos donde se practica periodos de cultivos alternados con producción de empastadas, los que pueden o no ser terrenos de aptitud agrícola; renoval denso, corresponde al bosque nativo secundario, con una cobertura de árboles superior al 75\%; matorral abierto, donde existe una cobertura de árboles menor al 25\% y una cobertura de arbustos entre 25 y $50 \%$; plantación, que corresponde a especies forestales exóticas o nativas plantadas, donde el estrato arbóreo es el dominante; matorral denso, matorral donde su 
cobertura es mayor a 75\%; y matorral arborescente semidenso, con árboles mayores a 2 metros de altura y con una cobertura de arbustos entre 50 y $75 \%$ (CONAF \& CONAMA, 1995).

La comunidad indígena Zewko comprende un total de 60 familias con un promedio de 5 personas cada una, es decir posee aproximadamente una población de 300 personas; en donde la superficie media familiar es de 6 hás. la división predial entonces es elevada. Referente a la composición por sexo existe un predominio de población masculina $(53,8 \%)$ por sobre la femenina $(46,2 \%)$. En la comunidad, la religión mayormente profesada es la católica, presentando un $55 \%$ en comparación con la pentecostal, adventista y anglicana presentes también en ella (Durán et al., 2000).

La zona cuenta con una estación médico rural y con un establecimiento educativo particular subvencionado (Escuela Prat N³29), los cuales dependen del municipio de Nueva Imperial.

(www.colegiosyliceosdechile.cl/)

\section{METODOLOGÍA}

La metodología se estructuró en procedimientos paralelos, los cuales se establecieron según la metodología convencional de caracterización botánica (florístico-vegetacional) y según metodologías de las ciencias sociales, teniendo presente que la investigación es de tipo exploratoria, lo que según Hernández et al., (1998) corresponde a un estudio donde el objetivo es examinar un tema o problema de investigación poco estudiado o que no ha sido abordado antes.

Con respecto a la metodología de las ciencias sociales, las técnicas de recolección de datos empleadas fueron: entrevistas abiertas semiestructuradas, reuniones grupales y visita a los ecosistemas elegidos. Las entrevistas fueron aplicadas a un grupo de 25 personas de la comunidad, que poseían mayor nivel de conocimiento del medio y la cultura. Desde la perspectiva metodológica, esta investigación se planteó respetar un protocolo en la relación con la comunidad. Para ello se estableció un acuerdo con los dirigentes y miembros de la comunidad, a quienes se presentó la iniciativa de investigación, sus objetivos, metodología y utilidad, Ilegando a un acuerdo para su realización. Los resultados de la investigación se fueron discutiendo a través del proceso de ésta con los dirigentes y la comunidad.

La técnica de análisis de datos que se utilizó fue la de análisis de contenido, la que según Krippendorff (1997) corresponde a una técnica de investigación destinada a formular, a partir de ciertos datos, inferencias reproducibles y válidas que puedan aplicarse a un contexto.

El análisis de las entrevistas se efectuó por medio de la codificación, proceso donde las características más relevantes del contenido del mensaje fueron transformadas a unidades que permitieron su descripción y análisis preciso.

En primer término se definieron las unidades de análisis, las que según Hernández et al., (1998) son segmentos del contenido de los mensajes que son caracterizados para ubicarlos dentro de categorías; las que a su vez son "casillas o cajones» en las cuales se clasifican las unidades de análisis (Holsti 1968, en Hernández et al., 1998).

Posteriormente, se elaboraron las hojas de codificación las cuales contienen las categorías y donde se anotó cada vez que una unidad entró en una categoría o subcategoría, lo que implicó contar las frecuencias de repetición de éstas y luego vaciar los datos de las hojas de codificación y obtener totales para cada categoría.

Referente a la metodología convencional de caracterización botánica, ésta se realizó mediante la delimitación de inventarios fitosociológicos que permiten determinar asociaciones vegetales, de acuerdo a la metódica europea (Braun-Blanquet, 1964), con una superficie para bosque de $100 \mathrm{~m}^{2}$, para matorral de $25 \mathrm{~m}^{2}$ y pradera de $4 \mathrm{~m}^{2}$, superiores al área mínima, entendida ésta como el área más pequeña en la cual la composición de especies está adecuadamente representada; identificación y registro de especies por parcela y realización de colectas intensivas en el área de estudio. Seguidamente se realizó el procedimiento de tabulación y se elaboró el catálogo florístico de acuerdo a Ramírez \& Westermeier (1973). 


\section{RESULTADOS}

\section{Ámbito sociocultural}

Desde el punto de vista de los comuneros, se identificaron 23 hábitats naturales, de los cuales el menoko (18\%), el malliñ (16,3\%) y el lil $(10 \%)$ se presentan como los mayormente reconocidos, en relación a la frecuencia con que fueron mencionados (Fig. 2).

Referente a los 8 hábitats más reconocidos (Fig. $3)$, ahora el menoko (38\%), el malliñ (19\%) y el trayen (13\%) son los que presentan los más altos porcentajes, en relación a la valoración sacra y de uso.

La Tabla 1 resume los espacios identificados mayoritariamente por la comunidad y a la vez los compara con los ecosistemas convencionalmente reconocidos.

La caracterización detallada de cada uno de los 23 hábitats naturales reconocidos se presenta a continuación, incluyendo además su ubicación y uso:

- Chozkin-mawiza: es un bosque pequeño (renoval), ubicado en los cerros. Se determinó sin uso, ya que se considera como reserva para las futuras generaciones.

- Malliñ: espacio plano inundado por largos periodos del año y altos niveles de humedad en verano, donde hay presencia de junquillos (Juncus spp.) y gran cantidad de plantas medicinales, se asocia este hábitat con la existencia del ngen. El uso que le asocian es mayormente medicinal. Entre las especies presentes en este espacio se encuentran el junquillo (Juncus procerus), menta (Mentha piperita), poleo (Mentha pulegium), paja ratonera (Anthoxanthum utriculatum), chépica (Agrostis capillaris) y contrayerba (Gratiola peruviana).

- Malliñko: laguna ubicada en zonas bajas. Corresponde a un estado inundado del malliñ.

- Mawiza o mawizantu: es un bosque nativo adulto ubicado en los montes o cerros de gran altura, las especies que predominan son el roble (Nothofagus obliqua), canelo, boldo (Peumus boldus) y laurel
(Laurelia sempervirens). El uso que hoy se le da a este espacio es la extracción de plantas medicinales, siembra de trigo, extracción de leña para la venta, la construcción o la producción de carbón de hualle y refugio de animales. Actualmente el espacio está muy deteriorado ya que se ha talado y reemplazado por pino (Pinus radiata) y eucalipto (Eucalyptus globulus).

- Menoko: espacio donde existe vegetación nativa, preferentemente leñosa y por lo tanto muchas plantas medicinales; la presencia de agua durante todo el año está en directa relación con que este es un lugar de nacimiento de agua o vertiente. Según la perspectiva mapuche, es un espacio sagrado, donde hay que solicitar permiso para acceder a él, ya que existe una ngen-ko (dueño del agua) al cual se le debe pagar una especie de tributo. Este es un lugar de respeto y cuidado. El uso se orienta para la extracción de plantas medicinales realizada preferentemente por machi; aunque actualmente el espacio se utiliza para el abastecimiento de agua. Este espacio se puede encontrar ubicado en zonas altas y zonas bajas, y las especies que lo caracterizan son el temu (Blepharocalyx cruckshanksii), pitra (Myrceugenia exsucca), cortadera (Cyperus eragrostis), canelo (Drimys winteri), nalca, pilpil voqui (Cissus striata) y maqui (Aristotelia chilensis).

- Menokonto: lugar sagrado donde no se puede ingresar ya que existen fuerzas sobrenaturales. Es un espacio con abundante vegetación nativa (nalca, hualle o roble, maqui, temu). Se ubica sólo en zonas bajas y no tiene un uso determinado.

- Nalkanto: espacio donde prolifera mayormente la nalca; se ubica dentro del menoko y su uso es la extracción de la misma para la alimentación.

- Lil: es un espacio con mucha pendiente (aprox. $45^{\circ}$ ), el cual se presenta como corte o quebrada, donde conviven plantas medicinales como musgos, líquenes y helechos. Su uso es preferentemente medicinal.

- Lilkura: es un espacio también con mucha pendiente, pero se diferencia del anterior en que es rocoso o pedregoso, donde también existen plantas medicinales.

- Fotrako: espacio pantanoso donde hay presencia de árboles nativos. 
- Foyentu: espacio donde prolifera mayormente el foye (canelo) y se ubica dentro del menoko.

- Jozkontu: es un pantano con plantas medicinales ubicado en zonas altas o bajas.

- Jozkon o Jozako: lugar muy pantanoso donde el agua está estancada y tiene mínimo drenaje. Es un espacio que presenta ciertos riesgos, tanto para los humanos como para los animales. Se ubica en la vega y se usa preferentemente para la extracción de plantas medicinales. Entre las especies presentes están el junquillo, la cortadera, la paja ratonera, etc.

- Rulu: es un espacio plano, cercano a un curso de agua, que se utiliza para el cultivo.

- Trawmako: espacio pequeño con presencia de agua donde existen plantas medicinales, por lo que su uso preferencial es de esta índole. Se ubica en zonas altas. Referente a las especies que lo caracterizan se puede mencionar a la nalca (Gunnera tinctoria) y chilco (Fuchsia magellanica).

Trayen: es un espacio construido por el hombre, caracterizado también por la presencia de un salto de agua. Este espacio se utiliza para la extracción de agua tanto para la familia como para los animales.

Trayenko: es un espacio natural caracterizado por la presencia de una cascada o salto de agua, donde existen plantas medicinales como helechos, palo santo (Dasyphyllum diacanthoides), avellano (Gevuina avellana), radal (Lomatia hirsuta) y huilli-patagua (Citronella mucronata). En el sector Zewko las personas identificaron dos de estos espacios: küchaltue y küleng-küleng, lugares sagrados y ceremoniales donde se solicita bonanza cuando hay mal tiempo y se pide Iluvia cuando hay sequía, respectivamente.

- Wichillko: espacio con pendiente, por donde corre el agua desde los cerros.

- Wingkul: cerros con vegetación nativa; actualmente están libres de vegetación y por lo tanto se utilizan para el cultivo.

- Witrunko: es un estero donde existe vegetación nativa y abundantes plantas medicinales (boldo, maqui, chilco). Se ubica en zonas bajas y su uso es preferentemente medicinal.

. Wüfko: es una vertiente. Se refiere específicamente al cuerpo de agua.

En la Tabla 1 se resumen los ecosistemas reconocidos en forma mayoritaria por los mapuche y su paralelo correspondiente a la caracterización científico-occidental.

\section{ÁmBito cIENTífico - occidentAL}

\section{a) Vegetación}

Los resultados obtenidos de la caracterización vegetacional, realizada según la metodología de Braun-Blanquet (Tabla 2), resume dos asociaciones vegetales claramente diferenciadas por su estructura florística:

- Pradera húmeda de junquillo (Juncetum procerii Oberdorfer, 1960).

- Bosque pantanoso de temo y pitra (Blepharocalyo-Myrceugenietum exsuccae Oberdorfer, 1960).

b) Flora

El estudio florístico realizado en el sector Zewko, permitió identificar 91 especies de plantas vasculares, de las cuales su distribución taxonómica (Fig. 4) indica que el grupo mejor representado es el de las Dicotiledóneas (Magnoliopsida) con un 75,8\%, le siguen las Monocotiledóneas (Liliopsida) con un 18,6\%, existiendo sólo un 4,3\% de Pteridófitos y $1 \%$ de Gimnospermas. La Fig. 5 muestra que el porcentaje de especies nativas corresponde a $72 \%$, superando a las introducidas con un porcentaje de $27 \%$ y cosmopolitas con $1 \%$.

Con respecto a las formas de vida de la flora del sector, la mayor representación la tiene el grupo de los hemicriptófitos con un $40 \%$, seguido por los fanerófitos y nanofanerófitos con un $26 \%$ y $20 \%$ respectivamente, los 4 grupos restantes no alcanzan porcentajes elevados (Fig. 6). 
Con respecto al estado de conservación de las 91 especies registradas (Fig. 7), 89 de ellas se presentan fuera de peligro y sólo dos con problemas de conservación: el chupón (Greigia sphacelata) y lingue (Persea lingue), ambos en la categoría vulnerable.

\section{DISCUSIÓN}

El pueblo mapuche se caracteriza por ser una sociedad que ha interactuado regularmente con su entorno natural y ha generado conocimientos socioculturales en esa interrelación. Se puede afirmar que este conocimiento en su gran mayoría es producto del estrecho vínculo entre los distintos espacios de vida de la sociedad mapuche con su entorno geográficofísico (Quintriqueo et al., 1996).

El conocimiento que se genera a partir de este vínculo estrecho con su ambiente, pasa a formar parte del conjunto de creencias que es determinante en la vida cotidiana del ser mapuche. Así, de la totalidad de la población de Zewko sólo un 41\% manifestaron este conocimiento y le dieron valor al traspaso de las creencias de generación a generación. A este respecto, Melillán (1998) manifiesta que aunque el pueblo mapuche ha perdido o ha visto deteriorar su hábitat original en donde se desarrolla su cultura, sus relaciones familiares, su vida productiva y en general donde obtenía los distintos aspectos que le permitían existir como pueblo, aún perduran algunos elementos que la hacen ser una sociedad culturalmente diferenciada, que mantiene relaciones de respeto y protección con su medio ambiente y en general con todo lo que la rodea.

Según Quintriqueo et al. (1996), los distintos conocimientos elaborados con respecto a los elementos de la naturaleza (lof, mawiza, pülon, rupü, rukawe, menoko, lewfü) le permite al mapuche actuar y realizar distintas acciones socio-culturales y comunicarse con sus similares; en este sentido, la caracterización realizada en Zewko de los distintos espacios o elementos naturales que allí todavía prevalecen, favorece la mantención de los conocimientos tradicionales acerca de la naturaleza y con ello el natural vínculo con el medio ambiente.
El número de hábitats definidos, delimitados y caracterizados por parte de la comunidad Zewko ascendieron a 23, aunque algunos de ellos son más bien sinónimos, como el jozko con el jozako y el mawiza con el mawizantu. Desde el punto de vista científico-occidental, otros espacios también pueden considerarse sinónimos, como menoko y menokonto, lil y lilkura, malliñ y malliñko. Los espacios más reconocidos fueron el menoko con 38\%, malliñ con $19 \%$ y trayen $13 \%$. Al referirse a los usos que mayormente los comuneros les asignaron a los espacios, éstos fueron del ámbito medicinal, lo que concuerda con lo manifestado por Bragg et al. (1986) y Durán et al. (1997). Lo mismo ocurre con la connotación de sagrado de un espacio, el que está ligado al ámbito religioso dentro de la cultura mapuche, tema en el que estos espacios siguen siendo los más relevantes.

Desde el punto de vista vegetacional, sólo se pudo reconocer dos asociaciones vegetales nativas en el área de estudio, la pradera húmeda de junquillo (Juncetum procerii) y el bosque de temo y pitra (BlapharocalyoMyrceugenietum exsuccae), ya que por el alto grado de alteración de los espacios, no fue posible aplicar el método de Braun-Blanquet en todos ellos. Esto marca una clara diferencia con la caracterización mapuche, ya que la metodología de estudio en la concepción científico-occidental es fundamental a la hora de realizar una investigación. En este caso, por lo deteriorado de los ecosistemas en estudio y por no existir unidades homogéneas, no fue posible la aplicación del método en todos ellos.

La primera de las asociaciones identificadas es del tipo palustre o emergente, cuya especie dominante es el junquillo (Juncus procerus), la que de acuerdo a Ramírez et al. (1992) se formaría a causa de la acción humana, introduciendo ganado en zonas donde el bosque originario habría sido talado. Esta asociación corresponde al ecosistema o espacio mapuche denominado malliñ, y que como ya se mencionara es un proveedor importante de plantas medicinales, utilizándose también para el pastoreo de animales.

Por otra parte, está el bosque pantanoso de temo y pitra, que corresponde a una comunidad leñosa de tipo azonal que depende más de las condiciones de humedad edáfica que de otro factor ambiental 
(Ramírez, 1982; Ramírez et al., 1983 en Hauenstein et al., 2001). Ramírez et al. (op. cit.) se refiere a este bosque como una asociación que prospera en la depresión intermedia, y que corresponde a una comunidad boscosa siempreverde, de poca altura, pobre en especies y monoestratificada; que ocupa hondonadas con mayor humedad edáfica y riberas de cursos de agua. En este sentido, en Zewko esta asociación se presenta muy escasa y con altos niveles de deterioro debido a la constante presión antrópica ejercida en ella, con un bajo número de especies y relacionada también a algún cuerpo de agua. Esta comunidad corresponde al ecosistema mapuche llamado menoko, el que es importante como aportador de plantas medicinales y de leña.

Según Ramírez et al. (1992), en el valle del río Imperial, dicho bosque al ser cortado y sin la intervención humana se degrada en un matorral de quila que puede permitir la regeneración del bosque original una vez cumplido su ciclo natural de vida; situación que concuerda con lo observado en este estudio.

Sin embargo, además se registró la presencia de remanentes del bosque de roble-laurel-lingue altamente alterados; su presencia en los resultados no es producto de haber encontrado la asociación como tal, ya que como se mencionó anteriormente no se pudo utilizar la metodología de Braun-Blanquet, debido a que no existían unidades homogéneas y de un tamaño adecuado donde aplicarla. Su gran deterioro se debe con seguridad a su tala intensiva para la obtención de madera, leña y carbón. Los indicios de su presencia en el área de estudio se refieren a ejemplares aislados de las especies dominantes de la asociación, como son el roble (Nothofagus obliqua) y laurel (Laurelia sempervirens), lo cual es apoyado por Gajardo (1995), quien señala que en esta zona los bosques dominantes son: roblelaurel y lingue, olivillo y temo-pitra; por su parte Veblen \& Schlegel (1982) señalan que el despeje de los bosques transforma este tipo forestal en praderas $y$ campos cultivados, dominados por ejemplares dispersos y de gran tamaño. Asimismo, San Martín et al. (1991) especifican que este bosque ocupó toda la depresión central del centro-sur de Chile, entre las ciudades de Victoria y Puerto Montt.

Desde el punto de vista florístico, se identificaron 91 especies de plantas vasculares de las cuales 89 especies se presentaron fuera de peligro y sólo 2 con problemas de conservación. Sin embargo, independiente del porcentaje elevado de especies fuera de peligro, en el área sigue existiendo un gran deterioro del recurso vegetal, lo cual es ratificado por lo obtenido en el origen fitogeográfico y el espectro biológico. En este sentido, el origen fitogeográfico de las especies es un indicador para visualizar la acción antrópica sobre las comunidades vegetales (Hauenstein et al., 1988). En el área se encontró un porcentaje de especies nativas superior a las introducidas ( $72 \% \mathrm{~V} / \mathrm{S} 27 \%$ ), lo que estaría indicando una fuerte acción humana en el área, ya que de acuerdo a Ramírez \& San Martín (1991) un 25\% de especies introducidas en una comunidad demuestra que la intervención humana es alta. Las formas de vida o espectro biológico representan adaptaciones a condiciones térmicas e hídricas, que son los factores más importantes en los biotopos terrestres (Ramírez, 1988). La forma de vida mayormente representada en Zewko fueron los hemicriptófitos, que corresponden a hierbas perennes, generalmente malezas, las cuales abundan en climas templados y son indicadoras de intervención humana; esto señalaría la acción permanente que el hombre ha ejercido en el área de estudio. Además, Hauenstein et al. (1999) señalan que la abundancia de esta forma de vida indica la capacidad de soportar el pisoteo y pastoreo de animales, lo que sería una clara prueba de la sostenida acción humana sobre la comunidad.

Los resultados obtenidos en ambos ámbitos expresan una relación de fondo que confirma la importancia de asumir las investigaciones de una manera integrada, ya que existe una clara diferencia entre el sistema de conocimiento, clasificación y uso de los hábitats por los mapuche y el sistema ecológico occidental convencional, expresada en la forma de plantearse en el entorno natural. A este respecto, Toledo (2003) menciona el surgimiento en el último tiempo de disciplinas híbridas, tales como Ecología humana, Economía ambiental y Agroecología, entre otras, las que ya consideran la disonancia mencionada entre ambas culturas respecto de la visión del mundo.

Cardona (1987) manifiesta que una clasificación indígena normalmente tiene el fin de unir armoniosamente los diferentes conocimientos sobre el mundo en una teoría única. En cambio, en el pensamiento occidental, la clasificación del mundo 
tangible no está unida con la del mundo espiritual, y al contrario, se diferencia de ésta precisamente por un diferente criterio de comprobación. En una teoría indígena todo convive: animales, plantas, seres humanos vivos o muertos, seres terrenales o sobrenaturales.

En este sentido, cuando los habitantes de Zewko caracterizan sus espacios lo hacen según la forma que tienen de relacionarse con su entorno natural, se trata más bien de conocimientos que están arraigados a una convivencia cotidiana con estos espacios. En cambio, la caracterización científica occidental que se obtiene, se presenta objetivamente sobre estos ecosistemas, aportando información referente a asociaciones vegetales específicas presentes en el área estudiada, reconociendo su flora y el grado de deterioro existente.

Cuando un investigador trabaja aspectos tan distintos como son los socioculturales y científicos occidentales debe plantearse, en primer término, que es indispensable aprender a caminar entre dos mundos, ya que la formación de un cientista se sustenta en la comprobación de hechos a través de metodologías específicas, en cambio el trabajo en el ámbito sociocultural se enmarca en el conocimiento de un mundo no material donde la mayoría de las cosas se interiorizan a medida que se traspasan de generación en generación. La utilización de ambas concepciones en toda investigación potenciaría los resultados y conclusiones finales de éstas.

\section{CONCLUSIONES}

La comunidad de Zewko-Rüpükura reconoce 23 espacios o ecosistemas; de éstos, 8 revisten para ellos mayor importancia, de los cuales el menoko, el malliñ y el trayen son los que presentan los mayores porcentajes.

- Estos espacios representan lugares de múltiples usos, entre los que destacan el medicinal, ritual, alimenticio (cultivos), obtención de leña (carbón) y sin uso (reservas)

- La vegetación nativa del área se encuentra sometida a un alto grado de intervención antrópica y degradación, debido a la sobreutilización y reemplazo de los espacios ya subdivididos.
Aplicando el método de análisis de vegetación de Braun-Blanquet, sólo se identificaron con claridad dos asociaciones: el Juncal (Juncetum procerii) y el bosque de temo-pitra o pitranto (BlepharocalyoMyrceugenietum exsuccae). El primero de ellos corresponde a lo que la comunidad mapuche reconoce como malliñ, y el segundo, corresponde al menoko.

- En los espacios estudiados se registraron 91 especies de plantas vasculares, de las cuales el $72 \%$ son nativas, las formas de vida dominantes son los hemicriptófitos y sólo existen dos especies con problemas de conservación (vulnerables).

\section{AGRADECIMIENTOS}

Los autores agradecen el apoyo financiero prestado por el Proyecto IDRC $N^{\circ}$ 100091-002, y en forma muy especial a las familias de la comunidad Zewko participantes en esta investigación, por su tiempo, disponibilidad y aporte de sus conocimientos, lo que nos permitió acercarnos al mundo mapuche tan distinto al nuestro. También a Gabriel Llanquinao, profesor de la Facultad de Educación de la Universidad Católica de Temuco, que participó como facilitador intercultural.

\section{BIBLIOGRAFÍA}

BRAGG, K., E. HAUENSTEIN \& M. LATSAGUE (1986), "Transecto etnobotánico del sector mapuche". CUHSO, Chile 3(2): 57-75.

BRAUN-BLANQUET, J. (1964), PflanzensoziologieGrundzuge der Vegetationskunde. Springer Verlag, Wien. 665 pp.

CARDONA, G. (1987), Visión del mundo natural. Hombre y ambiente. Ediciones ABYA-YALA. Quito, Ecuador. 1(3): 9-43.

COHEN, E. (1976), "Environmental orientations: a multidimensional approach to social ecology". Current Antropology XIII. 
CONAF \& CONAMA (1995), Catastro y evaluación de recursos vegetacionales nativos de Chile. Manual de cartografía de la vegetación. Santiago, Chile. 108 pp.

DURÁN, T., J. QUIDEL \& E. HAUENSTEIN (1997), Conocimientos y vivencias de dos familias wenteche sobre medicina mapuche. Ediciones LOM y Universidad Católica de Temuco, Chile. 99 pp.

DURÁN, T., L. PERALTA, N. CARRASCO, G. LLANQUINAO, I. PÉREZ, G. KAISER, H. MORA, M. SÁNCHEZ \& A. UGEÑO (2000), Documento de trabajo: Proyecto Gestión de recursos ambientales mapuche. Proyecto IDRC Nº 100091-002. Chile. 148 pp.

GAJARDO, R. (1995), La vegetación natural de Chile. Clasificación y distribución geográfica. Editorial Universitaria. Santiago. 165 pp.

hauenstein, E., M. latsague \& D. CONTRERAS (1988), "Origen fitogeográfico y espectro biológico como medida del grado de intervención antrópica en comunidades vegetales". Medio Ambiente, Chile 9(1): 140-142.

HAUENSTEIN, E., M. GONZÁLEZ, L. LEIVA \& L. FALCÓN (1999), "Flora de macrófitos y bioindicadores del lago Budi (IX Región, Chile)". Gayana Botánica, Chile 56(1): 53-62.

hauenstein, E., A. MUÑOZ, F. PeÑA \& $M$. GONZÁLEZ (2001), "Bases para la conservación de los Humedales de la costa de Toltén (IX Región)". Informe Final Proyecto $N^{\circ}$ 99-4-04. Universidad Católica de Temuco. 45 pp.

HERNÁNDEZ, R., C. FERNÁNDEZ \& P. BATISTA (1998), Metodología de la investigación. Segunda edición. Mc Graw-Hill Interamericana Editores, S.A. de C.V. México. 501 pp.

HIDALGO, J., V. SCHIAPPACASSE, H. NIEMEYER, C. ALDUNATE \& P. MEGE (1996), Culturas indígenas. Etnografía. Volumen II. Editorial Andrés Bello. Santiago, Chile. 303 pp.

KRIPPENDORFF, K. (1997), Metodología de análisis de contenido. Teoría y práctica. Primera edición. Editorial PAIDÓS. Barcelona, España. 279 pp.
LINCOLN, R.J., G.A. BOXSHALL \& P.F. CLARK (1995), Diccionario de ecología, evolución y taxonomía. Fondo de Cultura Económica, México. 488 pp.

LONCON, E. \& C. MARTíNEZ (1999), Pewenche Kimün, relatos sobre sabiduría pewenche. Primera edición. Programa EIB, Siedes. Temuco. 92 pp.

MELILLÁN, M. (1998), Evaluación del proyecto recuperación ambiental y manejo de microcuencas en tres comunidades mapuche de la comuna de Nueva Imperial, IX Región. Tesis Escuela de Agronomía, Universidad Católica de Temuco, Chile. 67 pp.

MOPT (1992), Guía para la elaboración de estudios del medio físico. Ministerio de Obras Públicas y Transporte. Madrid, España. 809 pp.

NAIKIAI, V. (1987), El uso del ecosistema en el antiguo pueblo Shuar. Hombre y Ambiente. Ediciones ABYA-YALA. Quito, Ecuador 1(1): 7-30.

OBERDORFER, E. (1960), "Pflanzensoziologische Studien in Chile-Ein vergleich mit Europa". Flora et Vegetatio Mundi 2:1-208.

PEÑA, L. (1989), Apuntes de conservación de suelos. Ediciones Universitarias de Concepción. Chillán. 135 pp.

QUINTRIQUEO, S., B. ANCAVIL, N. CATRILAF, C. CURIHUINCA \& E. PAILLAN (1996), Proceso de construcción del conocimiento mapuche en relación a los wingkul y su socialización en la comunidad escuela. Universidad Católica de Temuco. Facultad de Educación. Pedagogía en Educación Básica Intercultural. Temuco, Chile. 109 pp.

RAMÍREZ, C. (1982), Pasado, presente y futuro: la vegetación nativa del sur de Chile. Creces, 3(6-7): 40-45.

RAMÍREZ, C. (1988), "Formas de vida, fitoclimas y formaciones vegetales". El Árbol... Nuestro Amigo. Temuco, Chile 4 (1): 33-37.

RAMíREZ, C. \& C. SAN MARTÍN (1991), "La transformación antrópica de la vegetación de los Nadis del área mapuche en el centro-sur de Chile". Actas del XII Congreso Nacional de Arqueología Chilena (1): 205-214 
RAMÍREZ, C. \& R. WESTERMEIER (1976), "Estudio de la vegetación espontánea del Jardín Botánico de la Universidad Austral de Chile (Valdivia), como ejemplo de tabulación fitosociológica". Agro Sur, Chile 4(2): 93-105.

RAMÍREZ, C., C. SAN MARTÍN, J. RAMÍREZ \& J. SAN MARTÍN (1992), "Estudio sinecológico de las praderas del valle del curso inferior del río Imperial (Cautín, Chile)". Ciencia e Investigación Agraria, Chile 19(3): 97-112.

SAN MARTÍN, C., C. RAMÍREZ, H. FIGUEROA \& N. OJEDA (1991), "Estudio sinecológico del bosque de roble-laurel-lingue del centro-sur de Chile". Bosque, Chile 12(2): 11-27.
TOLEDO, V..M. (2003), “Antropología y Ecología: historia de un romance". CUHSO, Chile 7(1): 55-62.

TOLEDO, V., J. CABALLERO, C. MAPES, N. BARRERA, A. ARGUETA \& M. NÚÑEZ (1980), Los Purépechas de Patzcuaro: una aproximación ecológica. América Indigenista. Instituto Indigenista Interamericano. Editorial Libros de México. Volumen XL N 1. Enero-marzo. México. 191 pp.

VEBLEN, T. \& F. SCHLEGEL (1982), "Reseña ecológica de los bosques del sur de Chile". Bosque, Chile (4)2: 73-115.

http://www. cantovivo.org/

www.colegiosyliceosdechile.cl/

http://mapahumano.fiestras.com/

\section{Tabla 1: Resumen y caracterización occidental de los hábitats reconocidos}

\section{mayoritariamente en Zewko.}

\begin{tabular}{|c|c|}
\hline Ecosistemas mapuche & Ecosistemas occidentales \\
\hline Malliñ & $\begin{array}{l}\text { Pradera húmeda de junquillo (Juncetum procerii), en diferentes grados de } \\
\text { alteración y muy utilizada para pastoreo. }\end{array}$ \\
\hline Menoko & Bosque de temo y pitra (Blepharocalyo-Myrceugenietum exssuccae). \\
\hline $\begin{array}{l}\text { Mawiza } \\
\text { Mawizantu }\end{array}$ & Bosque de roble-laurel-lingue (Nothofagus-Perseetum linguae) \\
\hline Lil & $\begin{array}{l}\text { Quebrada o peredón rocoso de fuerte pendiente, donde predominan } \\
\text { especies como helechos y matorrales como el maqui, corcolén (Azara } \\
\text { integrifolia) y mata ratones (Coriaria ruscifolia). Corresponde al Fuchsio- } \\
\text { Chusqueetum. }\end{array}$ \\
\hline $\begin{array}{l}\text { Trayen } \\
\text { Trayenko }\end{array}$ & Cascada o pequeña caída de agua, con abundante y variada vegetación. \\
\hline Nalkanto & Población vegetal (Gunneretum) donde existe mayor abundancia de nalca. \\
\hline Jozkon & $\begin{array}{l}\text { Hábitat donde su mayor característica es la alta presencia de humedad y } \\
\text { muy pobre drenaje, semejante a un Ñadi. }\end{array}$ \\
\hline Rulu & Terraza aluvial, relativamente plana y apta para cultivos. \\
\hline Wingkul & Cerro. \\
\hline
\end{tabular}




\section{Revista CUHSO volumen 8 no 1}

Tabla 2. Tabla fitosociológica final que separa las comunidades obtenidas en los 8 inventarios levantados en la comunidad Zewko ( $A=$ Juncetum procerii, $B=$ BlepharocalyoM yrceugeni etum exsuccae* : especies asociadas positivamente con $\mathrm{H}$ olcus lanatus. ** : especies asociadas positivamente con Luma apiculata).

\begin{tabular}{|c|c|c|c|c|c|c|c|c|}
\hline Asociaciones & \multicolumn{6}{|c|}{ A } & \multicolumn{2}{|c|}{ B } \\
\hline Especies / Inventarios & 1 & 2 & 3 & 4 & 5 & 6 & 7 & 8 \\
\hline Holcus lanatus * & 30 & 20 & 10 & 5 & 10 & 10 & 1 & \\
\hline Agrostis capillaris* & 5 & 5 & 1 & & 5 & 10 & & \\
\hline Hipochaeris radicata* & 1 & & 1 & 1 & 1 & & 1 & \\
\hline Hedyotis salzmannii* & & 5 & & 10 & 1 & 1 & & \\
\hline Mentha pulegium* & & & 5 & 10 & & 10 & & \\
\hline Myosotis palustris* & 5 & 1 & & & 5 & & & \\
\hline Gratiola peruviana* & 1 & 1 & & & & 1 & & \\
\hline \multicolumn{9}{|l|}{ Polygonum hydropiperoides* } \\
\hline Dichondra sericea* & & & & & 5 & 1 & & \\
\hline Carex sp. ${ }^{*}$ & & & 5 & 10 & & & 1 & \\
\hline Luma apiculata ** & & & & & & & 5 & 5 \\
\hline Drimys winteri** & & & & & & & 5 & 5 \\
\hline Fuchsia magellanica** & & & & & & & 1 & 5 \\
\hline Blechnum cordatum** & & & & & & & 1 & 5 \\
\hline Escallonia revoluta** & & & & & & & 1 & 5 \\
\hline Boquila trifoliolata** & & & & & & & 1 & 1 \\
\hline Blechnum mochaenum** & & & & & & & 1 & 1 \\
\hline Chusquea quila** & & & & & & & 10 & 10 \\
\hline Juncus procerus & 25 & 10 & 50 & 5 & 50 & 50 & 1 & \\
\hline Lotus uliginosus & 20 & 30 & 10 & 20 & 15 & 5 & 1 & \\
\hline Eleocharis rivularis & 10 & 20 & 15 & 25 & 10 & 5 & 1 & \\
\hline Myrceugenia exsucca & & & & & & & 30 & \\
\hline Sophora macrocarpa & & & & & & & 10 & \\
\hline Acrisione denticulata & & & & & & & & 5 \\
\hline Caldcluvia paniculata & & & & & & & & 5 \\
\hline Nothofagus obliqua & & & & & & & 5 & \\
\hline Peumus boldus & & & & & & & 5 & \\
\hline
\end{tabular}


Ademásen inv. 1: Callitrichepalustris +; inv. 2: Ranunculussp. +, Roripa nasturtium-aquaticum $+;$ inv. 3: Prunella vulgaris +, Verbena bonariensis,$+ G$ amochaeta sp. + ; inv. 5: Rubusconstrictus + ; inv. 6 : Rumex acetosella + , Rubus constrictus + ; inv. 7: Rumex acetosella + , Prunella vulgaris + , Salix viminalis +, Rubus constrictus + , Callitriche palustris + , G unnera tinctoria + , Cyperus eragrostis,+ U ncinia phleoides + , Cirsi um vulgare + , Cissusstriata,$+ \mathrm{H}$ ydrocotyle ran unculoides + , Ranunculusrepens +, Digitalispurpurea + , Lapageria rosea. + , Azara serrata +; inv. 8: Rubus constrictus + , Ranunculus sp + , Aristotelia chilensis + , Jovellana violacea + , Solanum sp. + , Adiantum chilense + , O urisia sp. + , Acaena ovalifolia + .

Figura 1. U bicación geográfica de la comunidad Zewko-Rüpükura.

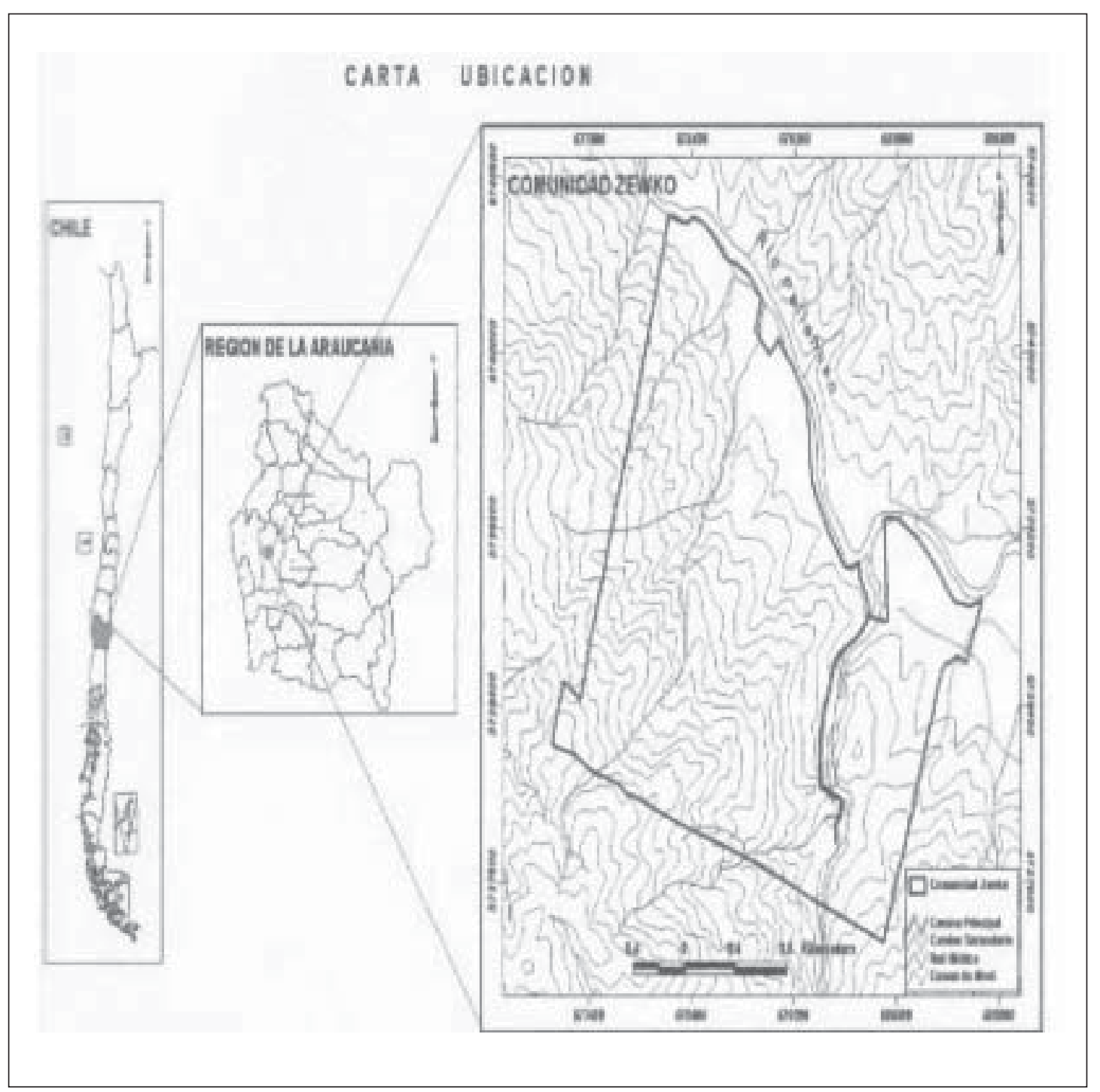



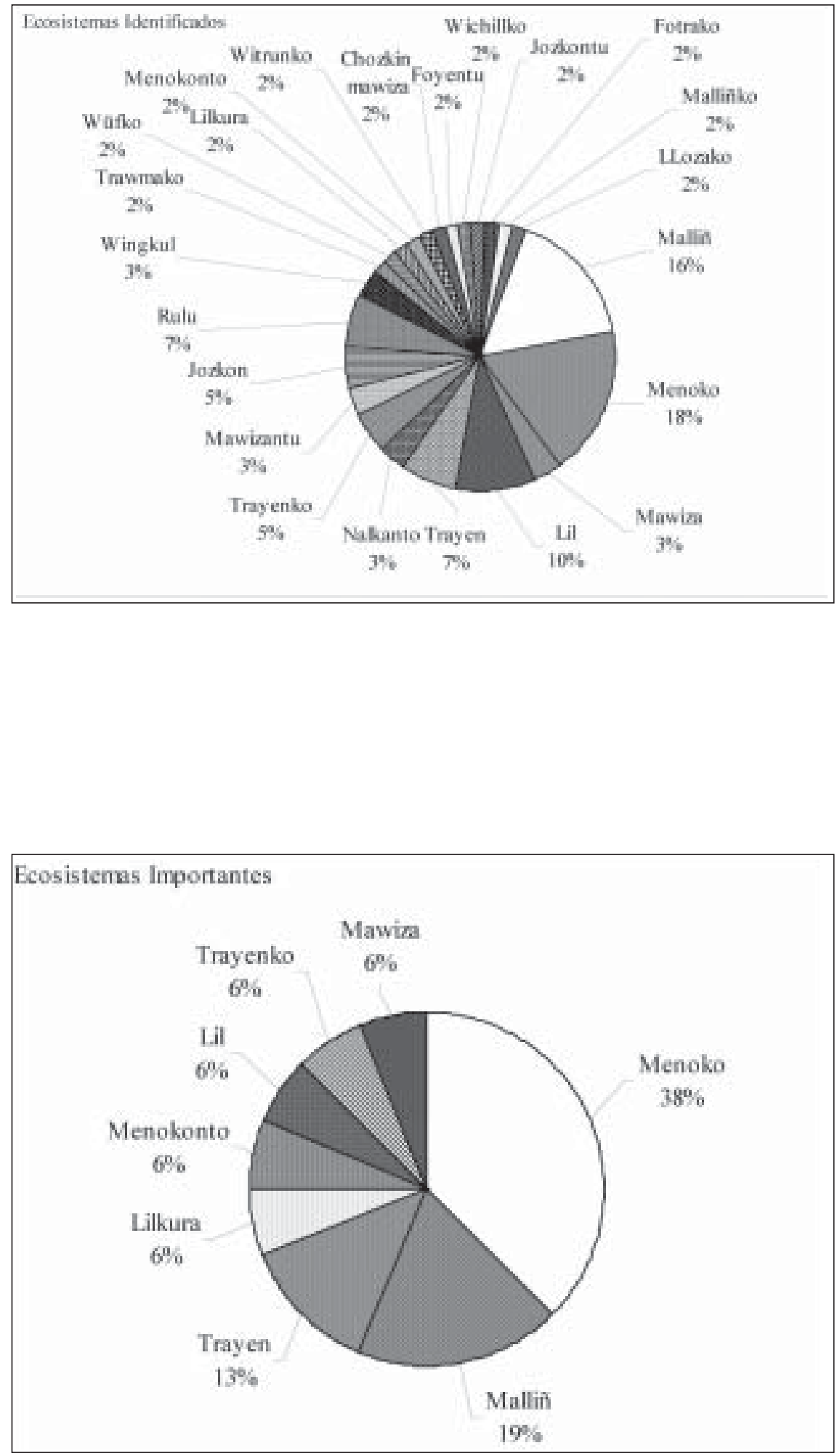

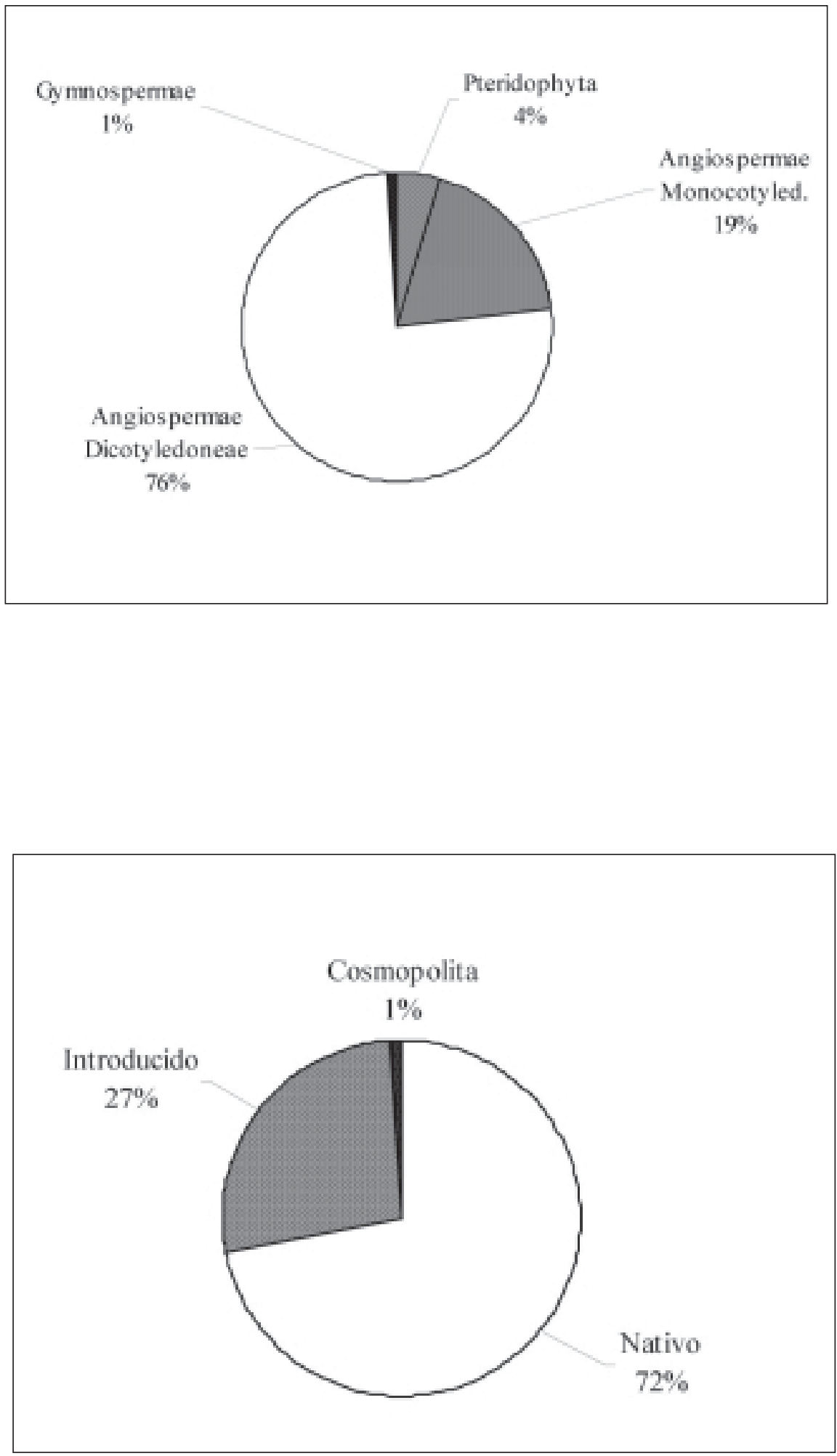

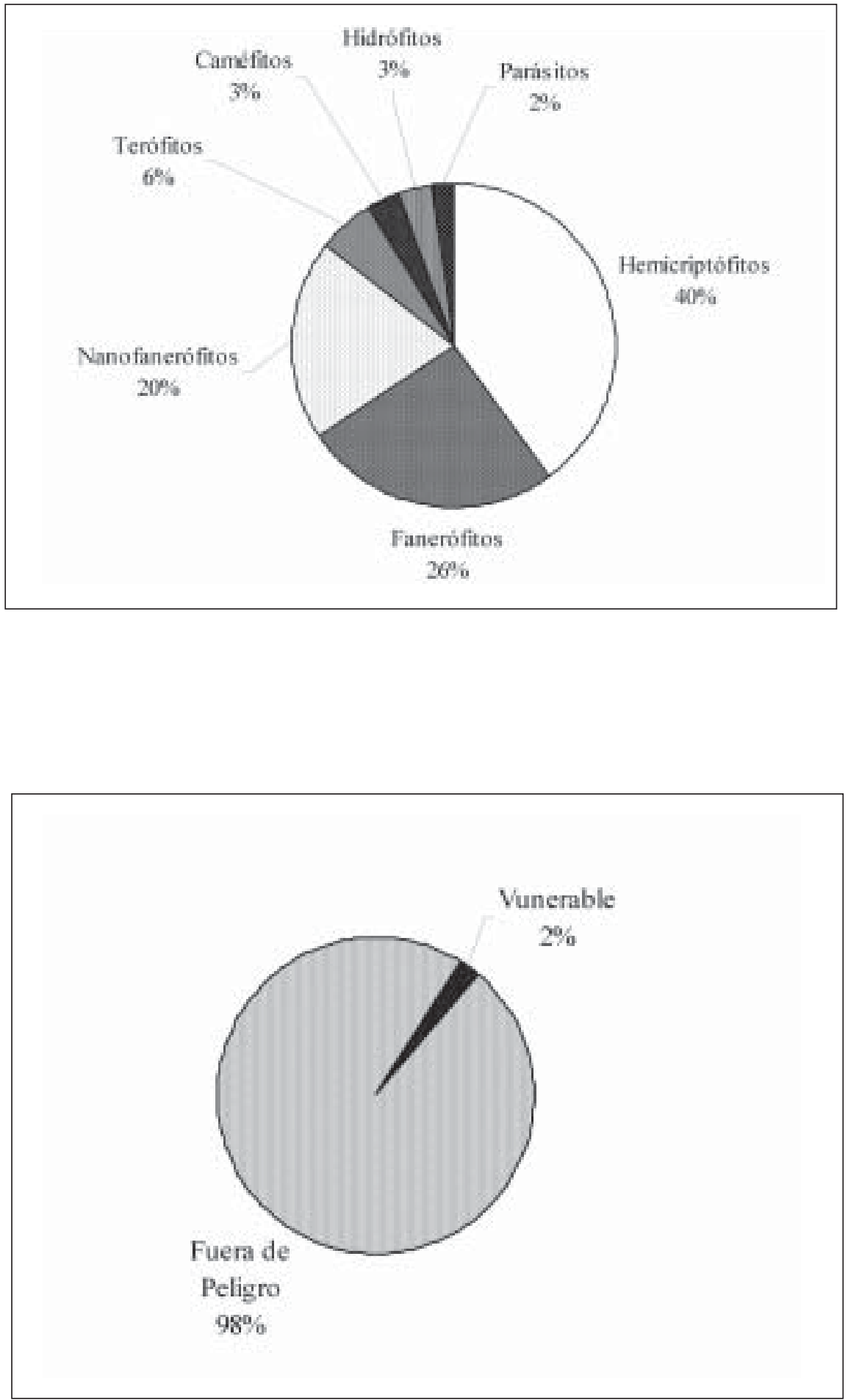